\title{
Strategy for detection of electrostatic spin-crossover effect in magnetic molecules
}

\author{
Aaron Hurley, Nadjib Baadji, and Stefano Sanvito \\ School of Physics and CRANN, Trinity College, Dublin 2, Ireland \\ (Received 17 April 2013; revised manuscript received 30 July 2013; published 12 August 2013)
}

\begin{abstract}
Scanning tunneling microscopy (STM) can be used to detect inelastic spin transitions in magnetic nanostructures comprising only a handful of atoms. Here we demonstrate theoretically that STM can uniquely identify the electrostatic spin-crossover effect, whereby the exchange interaction between two magnetic centers in a magnetic molecule changes sign as a function of an external electric field. The fingerprint of such effect is a large drop in the differential conductance as the bias increases. Crucially in the case of a magnetic dimer the spin-crossover transition inverts the order between the ground state and the first excited state, but does not change their symmetry. This means that at both sides of the conductance drop associated to the spin-crossover transition there are two inelastic transitions between the same states. The corresponding conductance steps split identically in a magnetic field and provide a unique way to identify the electrostatic spin crossover.
\end{abstract}

DOI: 10.1103/PhysRevB.88.054409 PACS number(s): 82.37.Gk, 72.25.-b, 73.20.-r, 75.76.+j

\section{INTRODUCTION}

Probing and manipulating spins in solid-state systems underpin the future development of spintronics and quantum information technology. In particular the interaction between conduction electrons and transition metal atoms with partially filled $d$ shells drives many low-temperature spin effects. Analyzing the conductance spectra of magnetic atoms adsorbed on a metallic host and probed by a scanning tunneling microscope (STM) unearths various distinctive features, which are indicative of many-body scattering. These include conductance steps associated to spin-flip transitions and zero-bias Kondo resonances. Such probing method, called spin-flip inelastic electron tunneling spectroscopy (SP-IETS), allows one to extract microscopic information about the spin interaction at the single atom level. ${ }^{1-9}$

So far SP-IETS experiments have all been well explained by assuming that the low-energy spin Hamiltonian for the atoms to probe is insensitive of the current or the STM voltage. Yet, recently it has been theoretically demonstrated that an electrostatic potential of sufficient strength can alter the exchange interaction between magnetically coupled atoms. ${ }^{10-13}$ In particular, it was shown that the magnitude of the energy Stark shift in a molecule containing two magnetic centers depends on whether the two spins are either parallel (high-spin) or antiparallel (low-spin) to each other, leading to a quadratic dependence of the exchange interaction between the two centers on the applied bias voltage. ${ }^{10}$ Furthermore, when an electrical dipole breaks the molecule inversion symmetry, the spin crossover may occur at experimentally achievable electric fields. This effect paves the way for the development of quantum information and spintronics devices. ${ }^{14}$ Preliminary experimental evidence of such electrostatic spin-crossover effect (ESCE) has been already provided in breaking junctions experiments with organometallic molecules. ${ }^{15}$ These experiments, however, are complex since the molecule can access both different spin and charging states.

Here we investigate the possibility of using STM to detect the dependence of the exchange coupling on an electrical potential, by using our previously developed quantum mechanical approach based on the nonequilibrium Green's function formalism. ${ }^{16-18}$ In particular, we calculate the biasdependent conductance spectra of an ideal molecule comprising two exchange-coupled spin 1/2 atoms. Previous attempts to investigate the ESCE have used a classical description of the localized spins, ${ }^{19}$ where quantized excitations were neglected. In contrast our fully quantum mechanical approach allows us to study in detail the elementary excitations of the system and identify the fingerprint of the ESCE. We will show that there exists a critical voltage, $V_{\mathrm{C}}$, where the conductance profile changes drastically and that this corresponds to the spin crossover between a low- and a high-spin state. Importantly, as the spin-crossover transition reverses the order between the ground state and the first excited state but not their symmetry, at both side of $V_{\mathrm{C}}$ there is an inelastic conductance step between spin states having same total spin. These split in the same way in a magnetic field and provide a unique way to identify the ESCE-driven transition.

\section{MODEL AND COMPUTATIONAL METHOD}

We consider a single-orbital tight-binding model, ${ }^{16-18}$ describing a magnetic system coupled to an STM tip and a substrate (sub). The scattering region containing the magnetic nanostructure consists of a magnetic dimer, where each atom $\lambda$ carries a quantum mechanical $\operatorname{spin} \mathbf{S}_{\lambda}$ and it is characterized by an on-site energy $\varepsilon_{0}$. We assume that the tip and the substrate can only couple to one atom at a time in the scattering region thus to broaden the electronic level $\varepsilon_{0}$ by $\Gamma_{\text {tip/sub }}\left(\Gamma=\Gamma_{\text {tip }}+\right.$ $\Gamma_{\text {sub }}$ is the total broadening). We model the spin-spin interaction between the localized spins by a nearest -neighbor Heisenberg Hamiltonian with coupling strength $J_{\mathrm{dd}}$. Furthermore, we include interaction with an external magnetic field $\mathbf{B}$. The electron-spin interaction Hamiltonian is constructed within the $s$ - $d$ model, ${ }^{20}$ where the transport electrons are locally exchange coupled to quantum spins through the exchange parameter $J_{\text {sd }}$. Thus, the full Hamiltonian for the magnetic nanostructure comprises three terms respectively describing the tight-binding electronic part $\left(H_{\mathrm{e}}\right)$, the spin part $\left(H_{\mathrm{sp}}\right)$, and 
the electron-spin interaction $\left(H_{\mathrm{e}-\mathrm{sp}}\right)$

$$
\begin{gathered}
H_{\mathrm{e}}=\varepsilon_{0} \sum_{\lambda \alpha} c_{\lambda \alpha}^{\dagger} c_{\lambda \alpha}, \\
H_{\mathrm{sp}}=2 J_{\mathrm{dd}}(V) \sum_{\lambda}^{N-1} \mathbf{S}_{\lambda} \cdot \mathbf{S}_{\lambda+1}+\sum_{\lambda}^{N} g \mu_{\mathrm{B}} \mathbf{B} \cdot \mathbf{S}_{\lambda}, \\
H_{\mathrm{e}-\mathrm{sp}}=J_{\mathrm{sd}} \sum_{\lambda \alpha, \alpha^{\prime}}\left(c_{\lambda \alpha}^{\dagger}\left[\sigma_{\lambda}\right]_{\alpha \alpha^{\prime}} c_{\lambda \alpha^{\prime}}\right) \cdot \mathbf{S}_{\lambda} .
\end{gathered}
$$

The electron ladder operators $c_{i \alpha}^{\dagger} / c_{i \alpha}$ create/annihilate an electron at site $i$ with spin $\alpha(\alpha=\uparrow, \downarrow), \mu_{\mathrm{B}}$ is the Bohr magneton and $g$ the gyromagnetic ratio. In Eq. (3) $\sigma$ is a vector of Pauli matrices.

The electron transport problem is solved by using the nonequilibrium Green's function scheme with a perturbative treatment of the electron-spin interaction, and where the perturbation parameter is $\alpha=J_{\text {sd }} / \rho$ ( $\rho$ is the electron density of states at the spin sites, see Refs. 16-18 for details). The diagonalization of Eq. (2) gives the eigenenergies, $\varepsilon_{n}$, and eigenstates, $|n\rangle$, of the spin system in the interacting region. Then, at the second order in the perturbation expansion, the transition rates, $W_{n l}$, between two eigenstates $|n\rangle$ and $|l\rangle$ induced by a non-spin-polarized current are given by

$$
W_{n l}=4 \frac{\rho J_{\mathrm{sd}}^{2}}{\Gamma} \sum_{i, \eta, \eta^{\prime}}\left|S_{n l}^{i}\right|^{2} \Gamma_{\eta} \Gamma_{\eta^{\prime}} \zeta\left(\mu_{\eta}-\mu_{\eta^{\prime}}-\Omega_{n l}\right),
$$

where the spin matrix elements $S_{n l}^{i}=\left\langle n\left|S^{i}\right| l\right\rangle$ with $i=$ $\{x, y, z\}$ are that of a single spin in the chain that is coupled to the tip (we drop the index $\lambda$ ). Furthermore $\zeta(x)=x /(1-$ $e^{-x / k_{\mathrm{B}} T}$ ) and $\mu_{\eta}$ is the chemical potential in $\eta$ th lead, $\eta=$ \{tip,sub\}. We also assume that the onsite energy is large enough for the density of states of the sample to remain constant in the small energy window of interest and therefore $\rho=\Gamma /\left(\varepsilon_{0}^{2}+\Gamma^{2}\right)$. The above transition rates can be used to evaluate the bias-dependent nonequilibrium population, $P_{n}$, of the spin states, $|n\rangle$, as the steady-state solution of the following equation

$$
\begin{aligned}
\frac{d P_{n}}{d t}= & \sum_{l}\left[P_{n}\left(1-P_{l}\right) W_{l n}-P_{l}\left(1-P_{n}\right) W_{n l}\right] \\
& +\left(P_{n}^{0}-P_{n}\right) / k_{\mathrm{B}} T,
\end{aligned}
$$

where $T$ is the temperature and $k_{\mathrm{B}}$ the Boltzmann constant (in the equation we take $\hbar=1)$. As $P_{n}$ enters in the definition of the Green's function for the transport electrons, Eq. (5) needs to be iterated self-consistently together with that of the electron propagator (see Ref. 16 for details).

At the second order the normalized current, I, flowing through a single spin can be expressed ${ }^{16}$ as a function of the potential bias, $V$, as

$$
I(V)=V+\frac{\rho J_{\mathrm{sd}}^{2}}{\Gamma} \sum_{i, m, k}\left|S_{n l}^{i}\right|^{2} P_{k}(V) i_{-}\left(V-\Omega_{m k}\right),
$$

where the normalization constant is given by the elastic conductance $G_{0}=\frac{2 e^{2}}{h}\left(\frac{\rho \Gamma_{\text {tip }} \Gamma_{\text {sub }}}{\Gamma}\right)$ and where we have defined the quantity $i_{-}\left(V-\Omega_{m k}\right)=\zeta\left(V-\Omega_{m k}\right)-\zeta\left(-V-\Omega_{m k}\right)$. In addition to the elastic contribution, the total normalized conductance, $G(V)=1+G_{1}(V)+G_{2}(V)$, includes two parts, respectively

$$
\begin{aligned}
G_{1}(V) & =\frac{\rho J_{\text {sd }}^{2}}{\Gamma} \sum_{i, m, k}\left|S_{n l}^{i}\right|^{2} P_{k}(V) \frac{d}{d V} i_{-}\left(V-\Omega_{m k}\right), \\
G_{2}(V) & =\frac{\rho J_{\mathrm{sd}}^{2}}{\Gamma} \sum_{i, m, k}\left|S_{n l}^{i}\right|^{2} i_{-}\left(V-\Omega_{m k}\right) \frac{d}{d V} P_{k}(V) .
\end{aligned}
$$

At equilibrium $P_{k}$ does not depend on $V$ and $G_{1}$ becomes the only contribution to $G(V)$. This provides a conductance step whenever the voltage coincides with inelastic energy transition $\Omega_{m k}=\left|\varepsilon_{m}-\varepsilon_{k}\right| / e$, being $e$ the electron charge. The conductance step height over the elastic conductance is then governed by the ratio $\rho J_{\text {sd }}^{2} / \Gamma$. However in the nonequilibrium case $P_{k}(V)$ is not constant and both $G_{1}$ and $G_{2}$ contribute, resulting in a more complicated nonlinear conductance profile.

\section{RESULTS AND DISCUSSION}

We now consider a model molecule undergoing the ESCE. This is described by a dimer of $S=1 / 2$ spins exchange coupled through the bias-dependent exchange parameter $J_{\mathrm{dd}}(V)=$ $J_{\mathrm{dd}}^{0}+b V^{2} \cdot{ }^{10}$ The parameter $J_{\mathrm{dd}}^{0}$ is the exchange in absence of an electric field, while $b$ defines the value of the critical voltage, $V_{\mathrm{C}}= \pm \sqrt{-J_{\mathrm{dd}}^{0} / b}$, at which the magnetic coupling switches from ferromagnetic $\left(J_{\mathrm{dd}}>0\right)$ to antiferromagnetic $\left(J_{\mathrm{dd}}<0\right)$. Here we consider, in line with density functional theory predictions, ${ }^{10} J_{\mathrm{dd}}^{0}=6 \mathrm{meV}$ and $b=-0.04 \mathrm{meV} /(\mathrm{mV})^{2}$, which give us $V_{\mathrm{C}} \sim 12.25 \mathrm{meV}$. The other parameters of the model are fixed either from experimental data or from theoretical considerations for systems presenting a similar STM setup. First, we consider an $s$ - $d$ exchange interaction $J_{\mathrm{sd}}=500 \mathrm{meV}$, as found from theory. ${ }^{21}$ We further assume that the electronic energies of the dimer atoms are of the order of $1 \mathrm{eV}$, far enough from the Fermi energy. This produces a constant density of states $\rho$ in the low-energy window of interest to the transport. The atoms are coupled to the underlying substrate strongly enough to produce a broadening of the onsite energies, $\Gamma_{\text {sub }}=250 \mathrm{meV}$. Furthermore, we assume that the tip is non-spin-polarized and movable such that conductance spectra can be investigated for a range of $\Gamma_{\text {tip. }}$. We will also examine the effect of magnetic fields on the spectra and fix the Lande $g$ factor to 2. All spectra are obtained at a temperature of $1.5 \mathrm{~K}$.

The top panel of Fig. 1 shows the total conductance calculated when the tip is positioned above one of the atoms forming the dimer, and the tip-to-atom coupling is weak, $\Gamma_{\text {tip }}=5 \mathrm{meV}$. In the same panel we show how the spectrum is decomposed into its various parts, $G_{1}$ and $G_{2}$ [see Eqs. (7) and (8)].

The conductance spectrum displays a first conductance step at about $5 \mathrm{mV}$, corresponding to the singlet to triplet spinexcitation. Then at the spin-crossover critical voltage, $V_{\mathrm{C}}$, there is a second step in the opposite direction, i.e., the conductance drastically drops. This feature can be easily understood by looking at the population of the spin states as a function of bias, also plotted in Fig. 1(b).

For $V<V_{\mathrm{C}}$ the molecule is in the singlet state, which is occupied with $P=1$. Note that the inelastic transition at $V \sim 5 \mathrm{mV}$ does not alter the spin population since the current is small and not spin polarized, so that it does not produce spin 


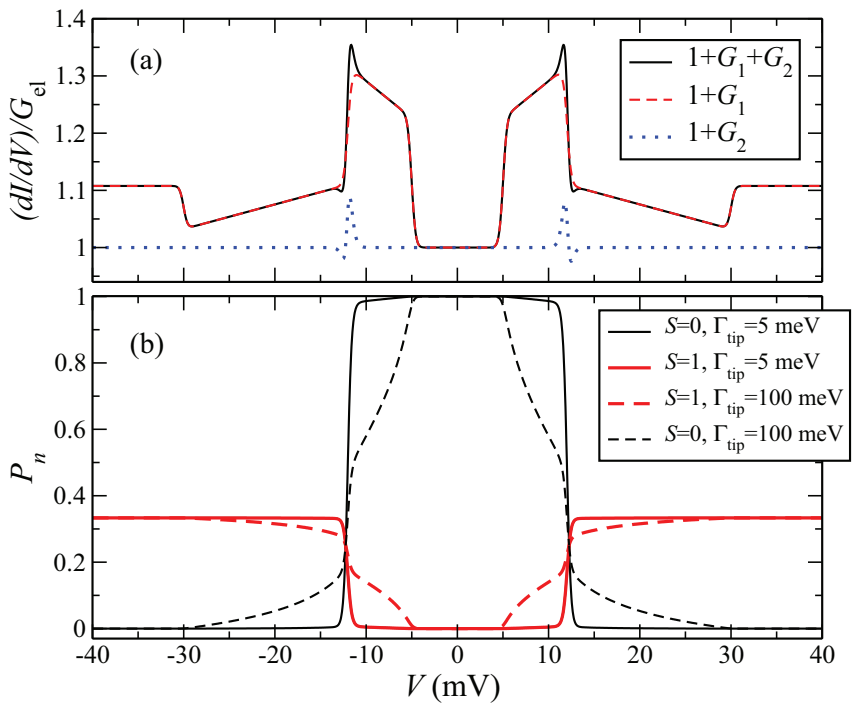

FIG. 1. (Color online) Conductance spectrum for a magnetic dimer exchanged coupled through a voltage-dependent exchange parameter $J_{\mathrm{dd}}$. (a) total normalized conductance and the different contributions to the total conductance. The conductance spectrum is calculated by assuming a week tip-to-atom electronic coupling, $\Gamma_{\text {tip }}=5 \mathrm{meV}$. Note the large dip in the conductance at $V_{\mathrm{C}} \sim 12 \mathrm{mV}$, corresponding to the spin-crossover transition. (b) Population of the singlet (black line) and triplet (red line) states for weak, $\Gamma_{\text {tip }}=5 \mathrm{meV}$ (solid line), and strong, $\Gamma_{\text {tip }}=100 \mathrm{meV}$ (dashed line), tip-to-atom coupling.

pumping. In other words the spin system always relaxes back to the ground state in between two inelastic scattering events. At the crossover voltage a change in the ground state occurs and the population of the singlet state rapidly decreases to zero, while that of the triplet states reaches $1 / 3$. Note that the triplet state is three times degenerate, meaning that each of the third component of the $S=1$ state are equally occupied (there is no magnetic anisotropy in the model). Finally, as the bias is further increased a third conductance step appears at about $30 \mathrm{meV}$. This is now related to an inelastic transition between the new triplet ground state and the first singlet excited state, i.e., between the same spin states responsible for the transition found at $5 \mathrm{mV}$, whose energy order is now reversed. Again there is no spin pumping and the spin population remains unaffected.

From the figure it is rather clear that most of the spectral features originate from $G_{1}$, which does not depend on the derivative of the spin population. The effect of $G_{2}$ is evident as an additional highly nonlinear contribution to the conductance at the spin-crossover voltage, brought about by the derivative of the population of the spin states.

We now investigate how the line shape changes as the STM tip is brought into closer contact to the molecule, and Fig. 2 reports $G(V)$ for values of $\Gamma_{\text {tip }}$ in the 5-250 meV range.

From the figure one can clearly see that the conductance profile gets drastically modified, in particular in the spectral region near the spin-crossover transition. Again the results can be understood by looking at the evolution of the spin population as a function of bias, plotted for $\Gamma_{\text {tip }}=100 \mathrm{meV}$ in Fig. 1(b). The most striking feature is that there is a significant

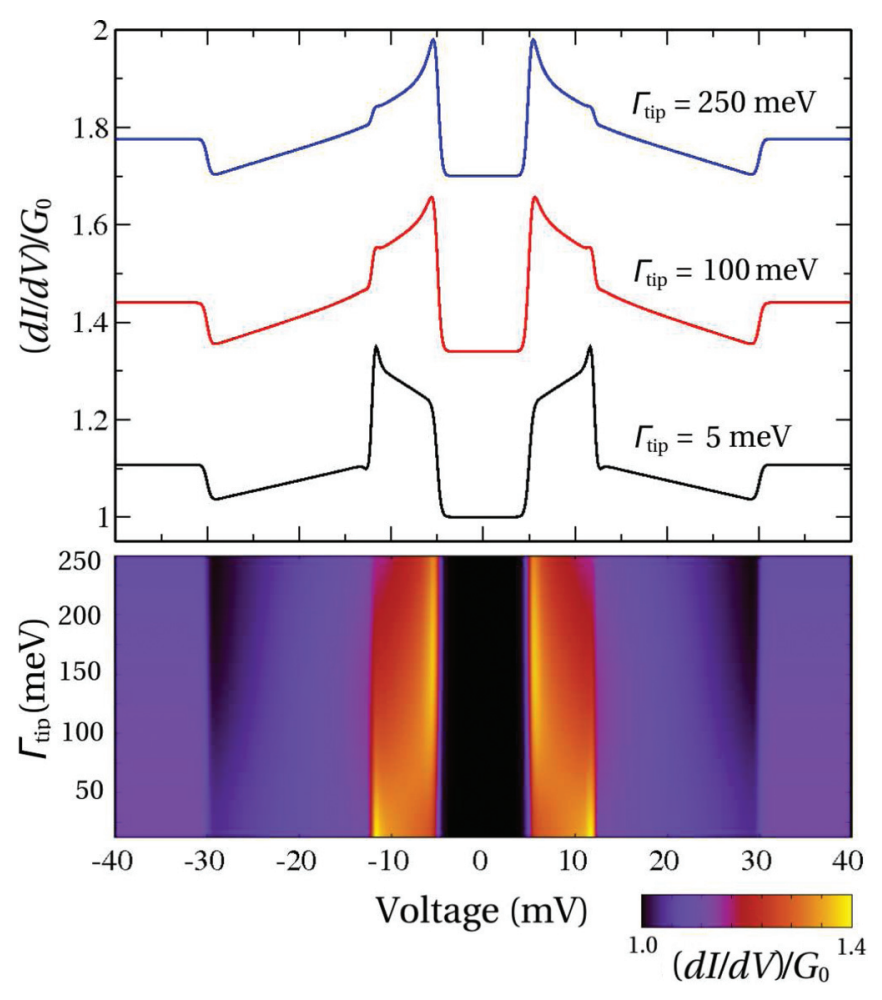

FIG. 2. (Color online) Conductance spectra for a spin $1 / 2$ dimer undergoing the ESCE. In the upper panel we show the normalized conductance spectra for three different values of $\Gamma_{\text {tip }}$, while the contour plot in the lower panel shows how the spectrum changes continuously between 0 and $250 \mathrm{mV}$. Note the weakening of the conductance drop at $V_{C}$ as the tip-to-atom coupling gets larger.

triplet (singlet) population for $V<V_{\mathrm{C}}\left(V<V_{\mathrm{C}}\right)$, i.e., that the more intense current is now able to maintain the molecule in a steady state in which both the ground state and the excited state are partially occupied. It is also not surprising that the mixing is maximized at around $V_{\mathrm{C}}$ since the exchange coupling is reduced and the two spin states are less energetically separated. This feature results in an enhancement of the first conductance step followed by the associated conductance decay. ${ }^{8,18}$

Finally we investigate the effects of applying a magnetic field and for the purpose of illustration we chose the rather large value of $20 \mathrm{~T}$ (at the limit of what is experimentally achievable). Figure 3 shows the evolution of the conductance spectrum as a function of $\Gamma_{\text {tip }}$. We notice that the onset of the magnetic field produces three equal sized and equally spaced conductance steps at around $5 \mathrm{mV}$. These originate from transitions between the $S=0$ ground state and the three Zeeman-split components of the $S=1$ excited state. Also in this case the spin-crossover transition produces a drastic drop in the conductance, which becomes less prominent as the tip-to-atom interaction strength gets larger. Intriguingly the same splitting occurs for the second conductance step at $\sim 30 \mathrm{mV}$, although in this case the Zeeman-split triplet states are resolved only for large tip-to-atom interaction. Note that, although for the inelastic transition at $5 \mathrm{mV}$ the initial state is $S=0$ and the final is $S=1$ while for the one at $30 \mathrm{mV}$ the order is reversed, in both cases the associated conductance step splits with an identical multiplicity. This is because the 


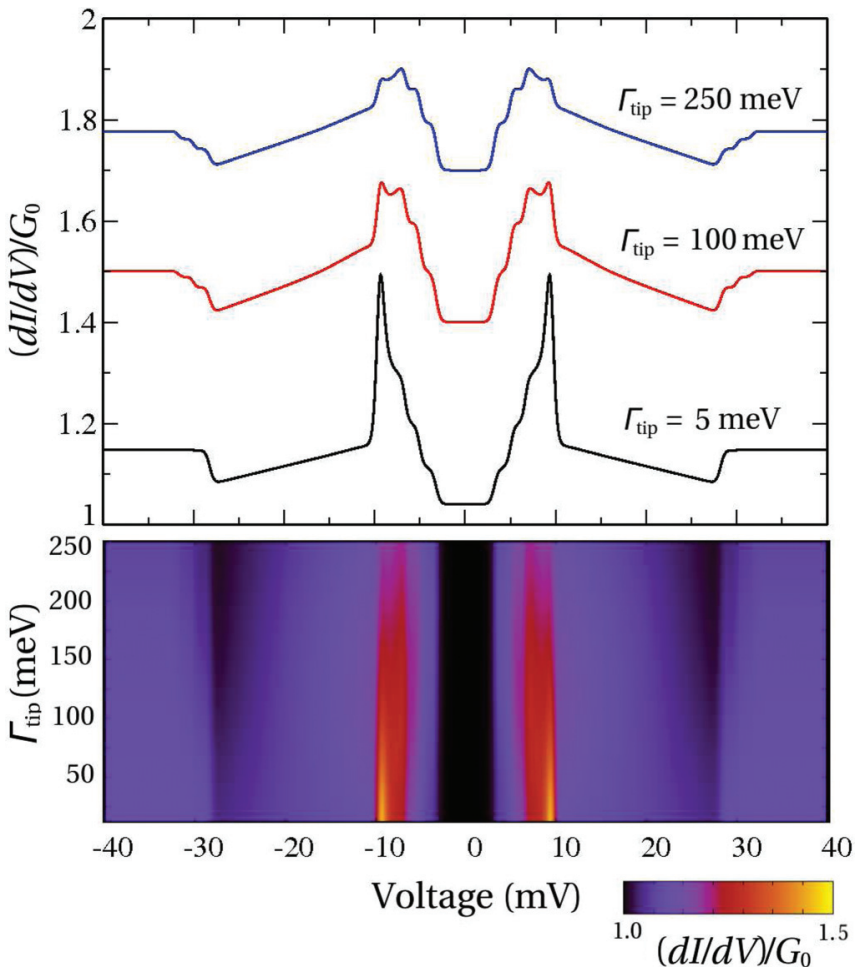

FIG. 3. (Color online) Conductance spectra for a spin $1 / 2$ dimer undergoing the ESCE and subject to a magnetic field of $20 \mathrm{~T}$. In the upper panel we show the normalized conductance spectra for three different values of $\Gamma_{\text {tip }}$, while the contour plot in the lower panel shows how the spectrum changes continuously between 0 and $250 \mathrm{mV}$. Note that the conductance steps corresponding to two inelastic transitions at both side of the spin-crossover transition split into three equally spaced and equally intense steps. This is due to the Zeeman splitting of the triplet state.

ESCE simply inverts the order of the two spin states involved in the transition but does not change their symmetry. Thus the presence in the conductance spectrum of a drastic drop located in between two inelastic transitions, which split identically in a magnetic field, is the fingerprint of the ESCE and provides a unique way for identifying it.

Note, however, that the situation can become sensibly more complicated if the spins of the two magnetic centers are not $S=1 / 2$. In general one still expects a well visible feature in the conductance trace at the spin-crossover voltage, but the excitation spectra at the two side of the transition will be in general different. Even more complexity is expected in the case where the molecule considered contains more than two magnetic centers. This time, in addition to a much more rich excitations spectrum, one has to face the fact that the voltage may be unevenly distributed across the molecule. This means that the actual spin-crossover voltage applied at the STM tip may not correspond directly to the one needed to make two spin states degenerate.

\section{CONCLUSION}

In conclusion we have investigated the possibility of detecting, by a spectroscopical STM experiment, the electrostatic spin-crossover effect, whereby the ground state of a molecule changes between singlet to triplet as the potential voltage increases. The hallmark of the spin-crossover transition is a drastic conductance drop, following the first conductance step associated to the singlet to triplet transition. Importantly as the ground state changes from singlet to triplet one expects a second step corresponding to the opposite transition (from triplet to singlet). One then has the situation in which the spin-crossover conductance drop is always placed in between two inelastic steps both associated to transitions between the singlet and the triplet. Such transitions can be easily identified by applying a magnetic field as Zeeman splitting resolves the step into three equally spaced and equally sized steps.

\section{ACKNOWLEDGMENTS}

This work is sponsored by the Science Foundation of Ireland (Grant No. 08/ERA/I1759) and CRANN. Computational resources have been provided by the Trinity Centre for High Performance Computing (TCHPC).
${ }^{1}$ C. F. Hirjibehedin, C. P. Lutz, and A. J. Heinrich, Science 312, 1021 (2006).

${ }^{2}$ C. F. Hirjibehedin, C.-Y. Lin, A. F. Otte, M. Ternes, C. P. Lutz, B. A. Jones, and A. J. Heinrich, Science 317, 1199 (2007).

${ }^{3}$ A. F. Otte, M. Ternes, K. von Bergmann, S. Loth, H. Brune, C. P. Lutz, C. F. Hirjibehedin, and A. J. Heinrich, Nature Phys. 4, 847 (2008).

${ }^{4}$ A. F. Otte, M. Ternes, S. Loth, C. P. Lutz, C. F. Hirjibehedin, and

A. J. Heinrich, Phys. Rev. Lett. 103, 107203 (2009).

${ }^{5}$ J. Fernandez-Rossier, Phys. Rev. Lett. 102, 256802 (2009).

${ }^{6}$ J. Fransson, O. Eriksson, and A. V. Balatsky, Phys. Rev. B 81, 115454 (2010).

${ }^{7}$ N. Lorente and J. P. Gauyacq, Phys. Rev. Lett. 103, 176601 (2009).

${ }^{8}$ B. Sothmann and J. Konig, New J. Phys. 12, 083028 (2010).
${ }^{9}$ R. Zitko, R. Peters, and Th. Pruschke, New J. Phys. 11, 053003 (2009).

${ }^{10}$ N. Baadji, M. Piacenza, T. Tugsuz, F. Della Sala, G. Maruccio, and S. Sanvito, Nature Mater. 8, 813 (2009).

${ }^{11}$ M. Diefenbach and K. S. Kim, Angew. Chem., Int. Ed. 46, 7640 (2007).

${ }^{12}$ M. Trif, F. Troiani, D. Stepanenko, and D. Loss, Phys. Rev. Lett. 101, 217201 (2008).

${ }^{13}$ A. Droghetti and S. Sanvito, Phys. Rev. Lett. 107, 047201 (2011).

${ }^{14}$ G. A. Timco, S. Carretta, F. Troiani, F. Tuna, R. J. Pritchard, C. A. Muryn, E. J. L. McInnes, A. Ghirri, A. Candini, P. Santini, G. Amoretti, M. Affronte, and R. E. P. Winpenny, Nature Nanotechnol. 4, 173 (2009). 
${ }^{15}$ V. Meded, A. Bagrets, K. Fink, R. Chandrasekar, M. Ruben, F. Evers, A. Bernand-Mantel, J. S. Seldenthuis, A. Beukman, and H. S. J. van der Zant, Phys. Rev. B 83, 245415 (2011).

${ }^{16}$ A. Hurley, N. Baadji, and S. Sanvito, Phys. Rev. B 84, 035427 (2011).

${ }^{17}$ A. Hurley, N. Baadji, and S. Sanvito, Phys. Rev. B 84, 115435 (2011).
${ }^{18}$ A. Hurley, N. Baadji, and S. Sanvito, Phys. Rev. B 86, 125411 (2012).

${ }^{19}$ S. K. Shukla and S. Sanvito, Phys. Rev. B 80, 184429 (2009).

${ }^{20}$ M. Stamenova, T. N. Todorov, and S. Sanvito, Phys. Rev. B 77, 054439 (2008)

${ }^{21}$ P. Lucignano, R. Mazzarello, A. Smogunov, M. Fabrizio, and E. Tosatti, Nature Mater. 8, 563 (2009). 